Philip Jan P. Arenga, MD

Joebert M. Villanueva, MD

Department of Otorhinolaryngology

Head and Neck Surgery

Western Visayas Medical Center
Correspondence: Dr. Joebert M. Villanueva Western Visayas Medical Center ENT Office

Q. Abeto St. Mandurriao, Iloilo City 5000

Philippines

Phone: (6333) 5090077

Fax: (6333) 3211797

Email: joebert_md@yahoo.com.ph

Reprints will not be available from the authors.

The authors declared that this represents original materia that is not being considered for publication or has not been published or accepted for publication elsewhere, in full or in part, in print or electronic media; that the manuscript has been read and approved by all the authors, that the requirements for authorship have been met by each author, and that each author believes that the manuscript represents honest work.

Disclosures: The authors signed disclosures that there are no financial or other (including personal) relationships, intellectual passion, political or religious beliefs, and institutional affiliations that might lead to a conflict of interest.

Presented at the Poster Session Contest on Surgical Innovation \& Instrumentation (1st Place), Philippine Society of Otolaryngology Head and Neck Surgery, Sofitel Philippine Plaza Hotel, CCP Complex, Manila, December 2, 2014.

\title{
The Use of a Soft Gel Capsule as a Medium for Modified Barium Esophagogram in Detecting Esophageal Foreign Body
}

\begin{abstract}
Objectives: To test a soft gel capsule with barium sulfate as a medium for modified barium esophagogram in detecting esophageal foreign body.
\end{abstract}

\section{Methods:}

Design: Preliminary Diagnostic Test Assessment; Consecutive Convenience Sample Setting: Tertiary Government Hospital

Patient: Soft gel capsule with barium sulfate was pilot tested on patients with a history and diagnosis of radiolucent foreign body ingestion between June 1 and November 30, 2014.

Results: Seven patients ( 6 males, 1 female; aged $26-61$ years) underwent the procedure. In all seven, the enhanced capsule immediately stopped above the level of the esophageal foreign body, easily identifying the exact location of the obstruction. Foreign bodies included 1 embryonated duck-egg white "balut", 5 chunks of pork meat and 1 claspless denture. All were successfully marked by the capsule on fluoroscopy and documented on X-ray. Esophagoscopy under general anesthesia was successfully performed after fluoroscopy in all patients.

Conclusion: We were able to improvise a new medium for use in modified barium esophagograms that was easy to prepare and that rendered good radiographic imaging and localization of radiolucent foreign bodies. A randomized trial in comparison to the prevailing test may confirm our findings further. Meanwhile, we recommend exploring the procedure in other hospitals as an alternative to barium-soaked cotton in the diagnosis of radiolucent esophageal foreign bodies

Keywords: soft gel capsule, barium suphate, esophagogram, esophageal foreign body

Esophageal foreign body obstruction is a common and potentially serious cause of morbidity among a wide spectrum of age groups..$^{1,2}$ In our setting, the most common esophageal foreign bodies are radiolucent (impacted meat or other food). ${ }^{3}$ Imaging including contrast studies are performed to identify the character and location of the foreign body and to rule out any complications. Biplane radiographs are the initial imaging modality of choice. Contrast examination is not recommended because of associated risk of aspiration and coating the foreign body or the mucosa precluding subsequent endoscopy. ${ }^{4}$ However, widespread local practice employs a cotton-ball coated in barium sulfate to diagnose radiolucent esophageal foreign bodies. As an alternative to cotton, we decided to test a soft gel capsule with barium sulfate as a medium for modified barium esophagogram in detecting esophageal foreign body. 


\section{METHODS}

Study Design preliminary diagnostic test assessment; consecutive convenience sample

Participants With technical review board approval, all patients with a history of foreign body ingestion consulting in the emergency room of the Western Visayas Medical Center (WVMC) between June 1 and November 30, 2014 were considered for this study. All those who were clinically diagnosed to have radiolucent foreign body ingestion and who gave informed consent was included. Patients diagnosed to have radiopaque foreign body ingestion were excluded.

\section{Preparation}

The materials used were:

1. Ibuprofen (Advil') Soft Gel Capsule (Pfizer Consumer Inc.; Zuellig, Philippines) (Figure1A \& 1B)
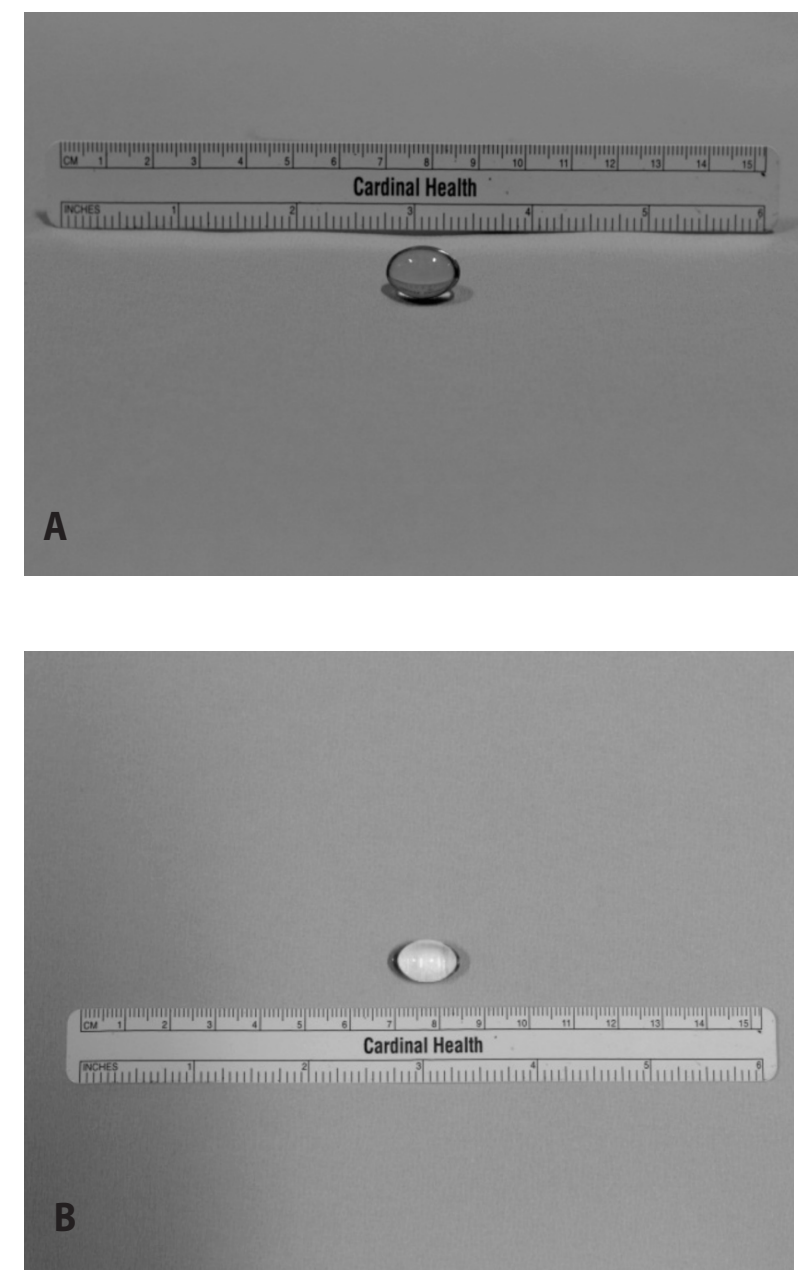

Figure 1A. Ibuprofen $\left(\mathrm{Advil}^{\odot}\right)$ Soft Gel Capsule before, and B. after replacing contents with barium sulphate solution.
2. Barium sulphate (E- Z-HD') $98 \mathrm{~g} / 100 \mathrm{~g}$ Powder for Suspension (Oral) Radiocontrast Media 340g (E-Z-EM, Inc., Anjou, Quebec, Canada) (Figure 1B)

3. Terumo $3 \mathrm{cc}$ syringe with gauge 23 needle (Terumo Philippines Corporation) (Figure 1C)

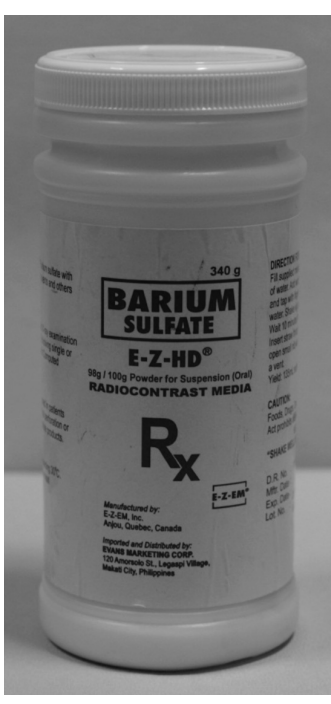

Figure 1B. Barium sulphate (E- Z-HD') $98 \mathrm{~g} / 100 \mathrm{~g}$ Powder for Suspension (Oral) Radiocontrast Media 340g (E-Z-EM, Inc., Anjou, Quebec, Canada)

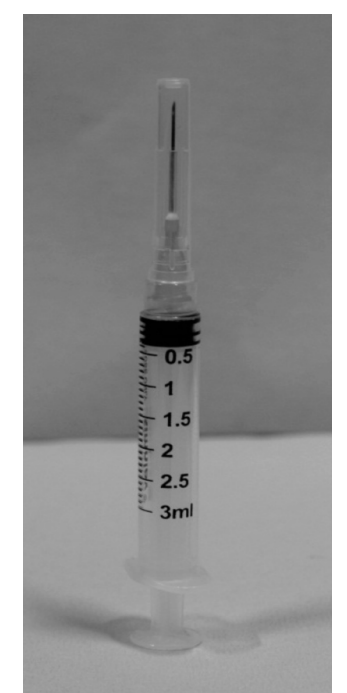

Figure $1 \mathrm{C}$. Terumo $3 \mathrm{cc}$ syringe with gauge 23 needle (Terumo Philippines Corporation)
4. Absolute pure distilled drinking water (Asia Brewery, Inc., Philippines)

Each soft gel capsule content was aspirated using a $3 c c$ syringe with gauge 23 syringe needle. A solution of 1:2 (barium sulphate: water) was injected back into the soft gel capsule using another syringe barrel with plunger into the same needle until the entire capsule was filled.

\section{Procedure}

Each patient donned a hospital gown was positioned standing for fluoroscopy using a Flexavision Collimator R-300 (Shimadzu Europa $\mathrm{GmbH}$, Dulsburg, Germany) at the WVMC radiology section and swallowed a prepared capsule with sips of water. As the soft gel capsule was ingested, it was tracked through the esophagus until it reached the area of obstruction. (Figure 2A) While the movement of the soft gel containing barium was being tracked on a fluoroscopic video monitor, a chest X-ray APL image was obtained using the same machine. (Figure 2B). Esophagoscopy under general anesthesia was done after fluoroscopy. 


\section{SURGICAL INNOVATIONS AND INSTRUMENTATION}

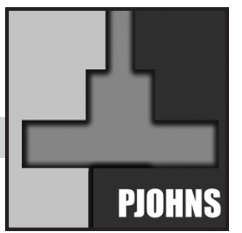

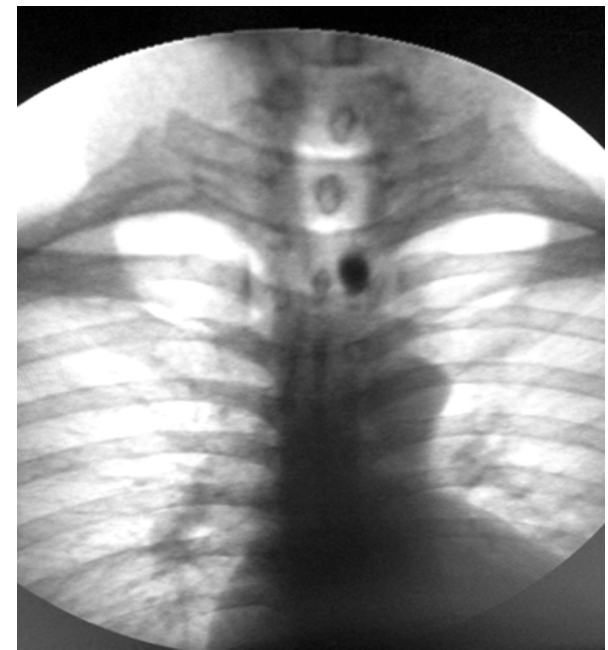

Figure 2A. Fluoroscopic study of a 56-year-old woman showing soft gel barium capsule as it was tracked through the esophagus until it reached the area of obstruction.

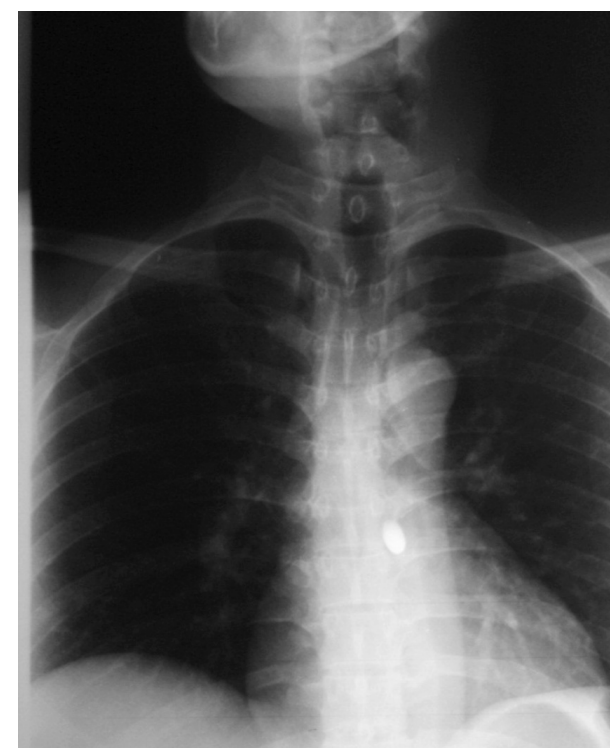

Figure 2B. Chest X Ray in a 56-year-old woman showing location of ingested meat marked by soft-gel barium capsule.

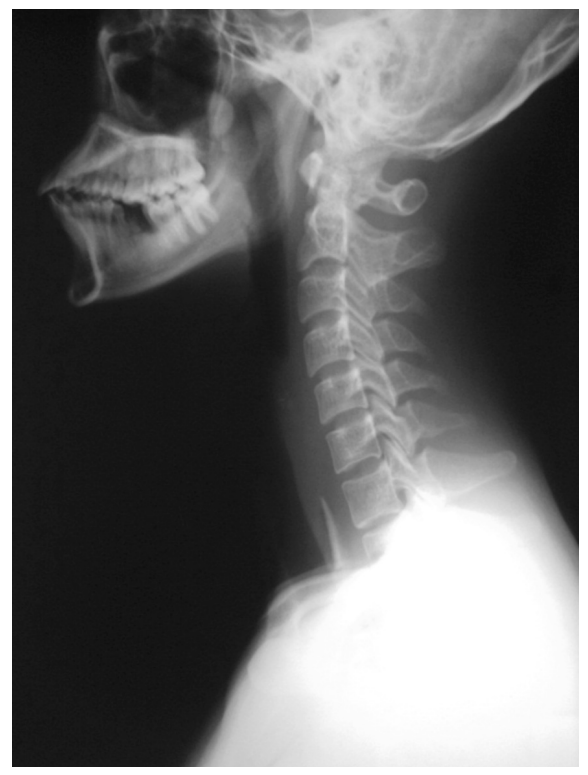

Figure 3A. Soft tissue lateral X Ray in a 29-year-old man showing location of ingested meat with bone.

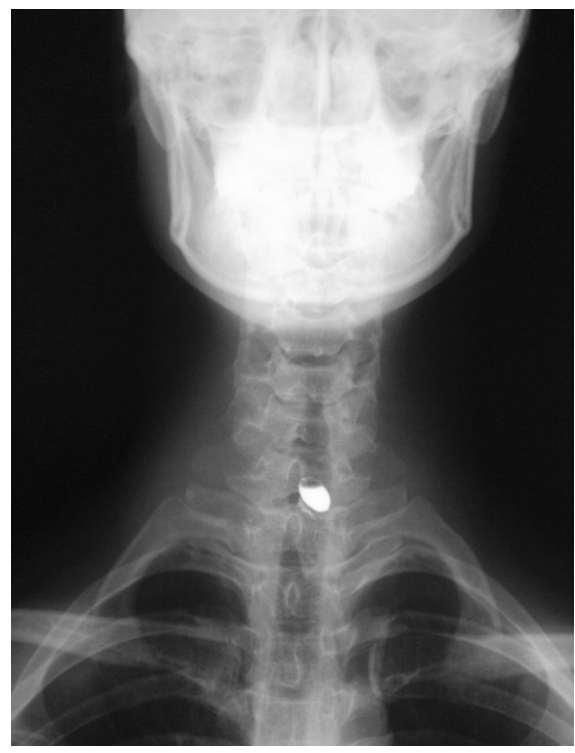

Figure 3B. Antero-posterior X Ray in a 29 year-old man showing location of ingested meat with bone

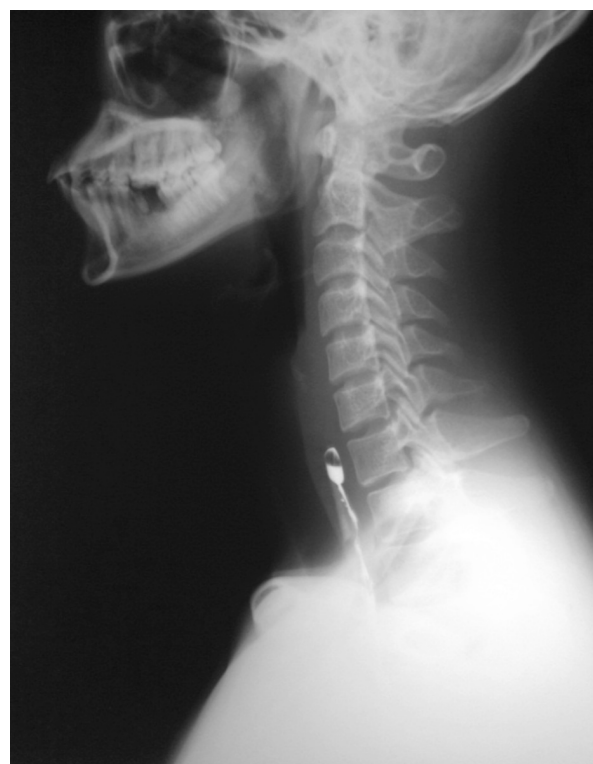

Figure 3C. Soft tissue lateral X Ray in a 29-year-old man showing location of ingested meat with bone marked by soft-gel barium capsule.

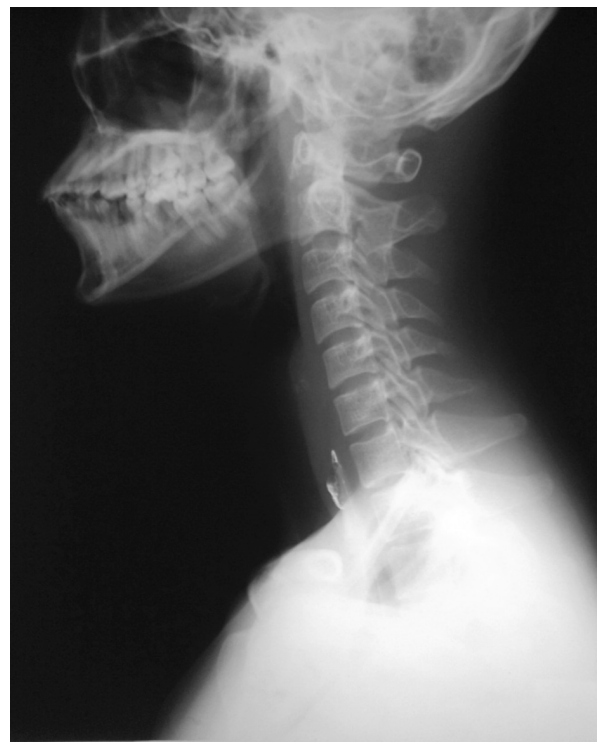

Figure 3D. Comparative image of soft tissue lateral $X$ Ray in a 29-year-old man showing location of ingested meat with bone marked by barium impregnated cotton. 


\section{RESULTS}

Seven (7) patients underwent the procedure, six (6) males and one (1) female with ages ranging from 26-61-years-old (mean age, 43-yearsold). In all seven, the enhanced capsule immediately stopped above the level of the esophageal foreign body easily identifying the exact location of the obstruction. The findings were one (1) embryonated duck-egg white "balut", five (5) chunks of pork meat and one (1) claspless denture. All were successfully marked by the capsule on fluoroscopy and documented on X-ray. (Figures $3 A-D$ ) In all 7 patients, esophagoscopy under general anesthesia was performed after fluoroscopy.

\section{DISCUSSION}

Accidental foreign body or large food bolus ingestion occurs primarily in edentulous, alcohol intoxicated or mentally impaired elderly subjects. ${ }^{5}$ At the Western Visayas Medical Center, food (typically meat and egg white of an embryonated duck egg or "balut") bolus impactions are the most common cause of esophageal foreign body obstruction in adults. It is hard to identify the exact location, presence or absence of a foreign body in the absence of bone or denture wires. ${ }^{6}$ Although endoscopy is of value in assessing gastrointestinal tract mucosal disease, barium studies are indispensable for clarifying uncertain findings at endoscopy or CT. However, barium studies are physically taxing, labor-intensive and difficult for radiologists to master. ${ }^{7}$ In our setting, a modified barium swallow involves the use of a cotton ball coated in barium sulfate solution which can be messy and uncomfortable for the patient as well.

In our study, all subjects swallowed the soft gel capsule without difficulty and expressed their ease in swallowing. We were also able to obtain a good radiographic view of the exact location of the foreign body in all cases. No adverse reactions were noted in any of the participants.

We were able to improvise a new medium for use in modified barium esophagograms that was easy to prepare and that rendered good radiographic imaging and localization of radiolucent foreign bodies. Our study only assessed our proposed diagnostic test in a preliminary manner, using a consecutive convenience sample without comparison to the prevailing test, and our results may not be applicable in all indicated situations. A randomized trial involving more subjects over a longer period in comparison to the prevailing test may confirm our findings further. Meanwhile, we recommend exploring the procedure in other hospitals as an alternative to barium-soaked cotton in the diagnosis of radiolucent esophageal foreign bodies.

\section{REFERENCES}

1. Gonzales DC, Gonzales RL. Esophageal foreign body impaction from ingested duck egg (balut) allantois: A case report. Research Folio [serial on internet]. 2000-2006 [cited 2015 May 12]. Available from: http://www.herdin.ph/index.php/component/herdin/?view=research\&ci $\mathrm{d}=1368$.

2. Abrenica RB, Chua AH. Esophageal and tracheobronchial foreign bodies: a ten year restrospective study. Philipp J Otolaryngol Head Neck Surg. 2004; 19(1-2): 33-40.

3. Ikenberry SO, Jue TL, Anderson MA, Appalaneni V, Banerjee S, Menachem T, et al. Management of ingested foreign bodies and food impactions. Gastrointest Endosc. 2011 Jun; 73(6): 10851091.

4. Libuit J, Banez V. Repeated foreign body ingestion in psychiatric patient. J Interv Gastroenterol. [serial on the internet] 2014 Oct-Dec; [cited 2014 Nov 20]; 4:4 [about 135-138 p.] Available from: http://www.jigjournal.org/sites/default/files/135-138\%20\%20JIG-2014.pdf.

5. Jackson CL. Foreign bodies in the esophagus. Am J Surg. [serial on the internet] 1957 Feb [cited 2014 Oct 2]; (2): [about 308-312p.]. Available from: http://www.americanjournalofsurgery.com/ article/0002-9610\%2857\%2990783-3/pdf

6. Palme CE, Lowinger D, Petersen AJ. Fish bones at the cricopharyngeus: a comparison of plain-film radiology and computed tomography. Laryngoscope. [serial on the internet] 1999 Dec [cited 2014 Oct 2]; 109(12): [about 1955-8p]. Available from: http://onlinelibrary.wiley.com/ doi/10.1097/00005537-199912000-00011/full.

7. Levine MS, Rubesin SE, Laufer I. Barium esophagography: a study for all seasons. Clin Gastroenterol Hepatol. [serial on the internet] 2008 Jan [cited 2014 Oct 2]; 6(1): [about 11-25p.] Available from: : http://www.cghjournal.org/article/S1542-3565\%2807\%2901058-0/fulltext

8. Hanes T. Information about soft gel ibuprofen. Livestrong.com. (serial on the internet) 2014 [updated 2013 Aug 16; cited 2014 Oct 1]. Available from: http://www.livestrong.com/ article/147723-information-about-soft-gel-ibuprofen/ 